\title{
Unsteady RANS Modeling of Flow around Two-Dimensional Rectangular Cylinders with Different Side Ratios at Reynolds Number $6.85 \times 10^{5}$
}

\author{
Shubiao Wang, ${ }^{1,2}$ Wenming Cheng, ${ }^{1,2}$ Run Du (D,, ${ }^{1,2}$ and Yupu Wang ${ }^{1,2}$ \\ ${ }^{1}$ School of Mechanical Engineering, Southwest Jiaotong University, Chengdu 610031, China \\ ${ }^{2}$ Technology and Equipment of Rail Transit Operation and Maintenance Key Laboratory of Sichuan Province, \\ Chengdu 610031, China
}

Correspondence should be addressed to Run Du; rdu@swjtu.edu.cn

Received 3 July 2020; Accepted 8 September 2020; Published 15 September 2020

Academic Editor: Zhengbiao Peng

Copyright ( 2020 Shubiao Wang et al. This is an open access article distributed under the Creative Commons Attribution License, which permits unrestricted use, distribution, and reproduction in any medium, provided the original work is properly cited.

In practical engineering, the Reynolds number $(R e)$ of box girder structure is usually very high $\left(\operatorname{Re} \geq 10^{5}\right)$, while most investigations of the flow around bluff bodies are concentrated on relatively lower Reynolds numbers (i.e., $R e=10^{3}-10^{4}$ ). This paper presented a numerical study of the unsteady flow around two-dimensional rectangular cylinders under a Reynolds number of $6.85 \times 10^{5}$ with different side ratios $(R=b / h$, width to height) ranging from 0.1 to 4.0 . Three unsteady Reynolds-averaged NavierStokes (RANS) two-equation $k-\varepsilon$ turbulence models (standard, RNG, and realizable) were adopted in the study. The realizable $k-\varepsilon$ model was chosen because it was found to perform the best among three models in the main aerodynamic integral parameters. According to the distinctions of aerodynamic characteristics with different side ratios, three regimes were divided and discussed in detail. The distribution of surface pressure over cylinders, the wake parameters, and vorticity contours of the rectangular cylinders with different side ratios were discussed.

\section{Introduction}

Bluff structures, which are the most common configurations in numerous practical applications such as tall buildings, bridges, chimneys, and fences, are of significance in both engineering and science when concerning the flow around them. Due to complicated fluid dynamic phenomena, such as separation, reattachment, and vortex shedding, the flow around bluff body has obtained much attractiveness.

The fundamental aerodynamic issues of bluff structures like square cylinders have been examined extensively in terms of numerical and experimental studies. Lyn et al. [1] studied the turbulent near-wake flow around a square cylinder at $R e=2.14 \times 10^{4}$ with two-component laser Doppler measurements. Shun et al. [2] discussed the flow structure, wake flow characteristics, and drag coefficient of a square cylinder at various $R e$ (ranging from 400 to $3.6 \times 10^{4}$ ) and incident angles (adjusting from $0^{\circ}$ to $45^{\circ}$ ) in an open-loop wind tunnel with the particle image velocimetry (PIV) technology. Rodi et al. [3] reviewed the Large Eddy Simulation (LES) and RANS methods for flow over a square cylinder and discussed the factors on the results such as the flow, grids, boundary conditions, and domain size. Sohankar [4] investigated the effects of the Reynolds number on results of the flow over a square cylinder using LES approaches and found that the effect is not much at higher $R e$, i.e., $\operatorname{Re}>2 \times 10^{4}$.

However, bluff bodies with rectangular cross section are employed more universally. Researches on rectangular cylinders' aerodynamic features and flow structures in detail are significant and meaningful for engineering practice. Many researchers have studied the flow around rectangular cylinders in experiments. Nakaguchi et al. [5] studied rectangular cylinders with different side ratios $(R=b / h$, where $b$ is the chord length along the direction of the flow and $h$ is the depth of the section) experimentally in a wind 
tunnel and found a peak in drag coefficient $\left(C_{d}\right)$ near $R=0.6$ and a discontinuity in Strouhal number $(S t)$ at $R=2.8$. Okajima et al. [6,7] studied the influence of $R$ on the lift and drag forces, base pressure, and $S t$ in the range of $R e$ between 70 and $2 \times 10^{4}$ experimentally and discovered a sudden change in $S t$ at $R=2.8$ and 6.0. Nakamura and Ohya [8] investigated the effect of scale and the intensity of turbulence on the wake of rectangles in a wind tunnel. They concluded that the small-scale turbulence could raise the growth rate of the separated shear layers and the large-scale turbulence could decline vortex shedding. Both scale and intensity of turbulence affect the mean base pressure $\left(-C_{p b}\right)$ apparently. Ohya et al. [9], Norberg [10], and Nakagawa et al. [11] also obtained similar results at different side ratios and Reynolds numbers.

Besides the experimental studies, numerical studies had also been carried out. Okajima et al. [12, 13] investigated flow characteristics of rectangular cylinders with $R=0.6-8$ by a finite difference method (FDM) at low $R e$ and by a discrete vortex method (DVM) at high $R e$. Their numerical results show a good agreement with their experimental ones $[6,7]$. Taylor et al. [14] studied the two-dimensional (2D) flow around cylinders with $R=0.25-3$ at $R e=2 \times 10^{4}$ using DVM. Their numerical methods were verified well with experimental results. Shimada et al. [15] analyzed the aerodynamic characteristics of cylinders with $R=0.6-8$ at $R e=2.2 \times 10^{4}$ numerically using a modified $k$ - $\varepsilon$ model. Their method presents better results than ordinary $2 \mathrm{D}$ analysis in vortex shedding, drag coefficients, and distributions of mean surface pressure. They reported a discontinuity in Strouhal number at both $R=2.8$ and $R=6$. They also explained that the reason of the underestimation in pressure and force fluctuations is that the stochastic components are not contained in RANS method. Tian et al. [16] demonstrated some inaccurate results when side ratio is lower $(R<0.6)$ using $k-\omega$ Shear Stress Transport (SST) method. Aerodynamics features of rectangular cylinders $(R=0.4-4)$ at $R e=10^{5}$ were simulated by Sohankar [17] using two LES approaches. The global integral results and the mean and instantaneous-flow structures were presented in detail in their study. Islam et al. [18] discussed these at $R e=100-250$ using incompressible lattice Boltzmann method (ILBM).

Although there have been many researches about the flow over rectangular cylinders before, they are mainly on relatively lower Reynolds numbers, e.g., experimental studies of Nakaguchi et al. [5] $\left(R e=6 \times 10^{4}\right)$, Bearman and Trueman [19] $\left(R e=2.2 \times 10^{4}\right)$, Mizota et al. [20] $\left(R e=2.1 \times 10^{5}\right)$, and Norberg [10] $\left(R e=1.3 \times 10^{4}\right)$ and numerical studies of Shimada and Ishihara [15] $\left(R e=2.2 \times 10^{4}\right)$, Sohankar [17] $\left(R e=10^{5}\right)$, and Tian et al. [16] $\left(R e=2.14 \times 10^{4}\right)$. Most of them were performed at $R e \leq 10^{5}$. However, the section size of the structure in practical applications (i.e., bridges, buildings, and engineering machineries) is usually larger, so the Reynolds numbers trend to be higher. For example, Re of the beam or leg component of the gantry or port cranes, which can be seen as the typical rectangular column structure, would be more than $5 \times 10^{5}$ when wind speed $(U)$ is $10 \mathrm{~m} / \mathrm{s}$ and when the characteristic height $(h)$ of them is designed to be more than $1 \mathrm{~m}$ (e.g.,
$1.998 \mathrm{~m}$ in [21] and $1.82 \mathrm{~m}$ in [22]). At some extreme cases ( $h=5-10 \mathrm{~m}, U \geq 30 \mathrm{~m} / \mathrm{s}$ ), the Reynolds number $R e$ would be up to $10^{7}$. As a result, it is necessary and meaningful to study the flow around rectangles with various $R$ when $R e$ is much higher. They are more common in engineering and could give some guidance for industrial design and manufacture.

In this paper, our main purpose is to study the flow around rectangular cylinders with different side ratios when Reynolds number is much higher. The main global parameters and mean and instantaneous-flow structures would be discussed in this work.

The rest of this paper is as follows. Section 2 gives a short presentation of numerical models, governing equations, boundary conditions, and grids. In Section 3, the results and discussion are presented, including the difference in different models, the effect of side ratios for global parameters, analysis of wake, and the vorticity contours. Some concluding remarks are given in Section 4.

\section{Numerical Methods}

Turbulence is a time-dependent three-dimensional process whose simulation by means of computational techniques is particularly challenging. Several turbulence models, comprising modified versions, have been proposed. They are different in their physical foundations, degree of complexity, and computer power demands. And the most commonly used models at present are the Reynolds-averaged Navier-Stokes (RANS) and Large Eddy Simulation (LES).

Rodi et al. [3], Murakami [23], Hanjalic [24], Menter and Florian [25], and Blocken [26] have done lots of investigations about the comparisons and discussions of these two methods. Although there is some difference between them, their basic views are relatively consistent. The LES approach applied in a 3D domain might be more accurate and reliable, but it is also the most computationally burdensome because of its high cost in wall boundary layers. It could not be widely used in industrial applications especially when Reynolds number is higher. And, as for the RANS models, due to the use of wall functions, the cost is less, and the accuracy of calculation is relatively acceptable; hence, it is still the most widely used turbulence model in engineering. Concerning a 3D flow domain, RANS models cannot resolve any other turbulence details except for single large-scale structures [25]; their results are practically similar to $2 \mathrm{D}$ simulations and show weak three dimensionalities $[27,28]$. That is, $3 \mathrm{D}$ simulations cost more but do not receive significantly better results than $2 \mathrm{D}$ ones for global parameters; thus 2D RANS is a better choice.

Taking into account the fact that the primary goal of this piece of research is the CFD-based computation of flow around rectangles with various side ratios in much higher $R e, 3 \mathrm{D}$ simulations have been discarded, and the computer cost linked with LES approaches could not be afforded by the authors; the 2D RANS $k-\varepsilon$ turbulence models based on the Boussinesq assumptions [29] are chosen. 
2.1. RANS $k$ - $\varepsilon$ Turbulence Model. The Reynolds-averaged incompressible Navier-Stokes equations [29] are expressed as

$$
\begin{aligned}
& \frac{\partial u_{i}}{\partial x_{i}}=0 \\
& \frac{\partial u_{i}}{\partial t}+u_{j} \frac{\partial u_{i}}{\partial x_{j}}=-\frac{1}{\rho} \frac{\partial p}{\partial x_{i}}+v \frac{\partial^{2} u_{i}}{\partial x_{j} \partial x_{j}}-\frac{\partial \overline{u_{i}^{\prime} u_{j}^{\prime}}}{\partial x_{j}},
\end{aligned}
$$

where $u_{i}$ is the velocity component in $x_{i}$ coordinate directions and, for the present $2 \mathrm{D}$ simulation, $i, j=1,2, x_{1}$ and $x_{2}$ are the streamwise and cross-stream directions, respectively; $p$ is the pressure; $\rho$ is the density of the flow; $t$ is the time; $\overline{u_{i}^{\prime}} u_{j}^{\prime}$ is the Reynolds stress component, where the superscript of $u_{i}$ is defined as the velocity fluctuation.

In the present study, the two-equation $k-\varepsilon$ turbulence models were adopted as our main methods. The $k-\varepsilon$ models which determine a turbulent length and time scale by solving two separate transport equations $k$ (the turbulence kinetic energy) and $\varepsilon$ (its rate of dissipation) are generally applied with three types: the standard, RNG, and realizable $k-\varepsilon$ models.

2.1.1. Standard $k-\varepsilon$ Model. The standard $k-\varepsilon$ model proposed by Launder and Spalding [30] contains two transport equations of $k$ and $\varepsilon$. The transport equation $k$ comes from the exact equation, but the transport equation $\varepsilon$ was gotten from physical reasoning and has little matter to its mathematically exact counterpart. And they are obtained from the following transport equations:

$$
\begin{aligned}
& \frac{\partial}{\partial t}(\rho k)+\frac{\partial}{\partial x_{i}}\left(\rho k u_{i}\right)=\frac{\partial}{\partial x_{j}}\left[\left(\mu+\frac{\mu_{t}}{\sigma_{k}}\right) \frac{\partial k}{\partial x_{j}}\right] \\
&+G_{k}+G_{b}-\rho \varepsilon-Y_{M}+S_{k}, \\
& \frac{\partial}{\partial t}(\rho \varepsilon)+\frac{\partial}{\partial x_{i}}\left(\rho \varepsilon u_{i}\right)=\frac{\partial}{\partial x_{j}}\left[\left(\mu+\frac{\mu_{t}}{\sigma_{\varepsilon}}\right) \frac{\partial \varepsilon}{\partial x_{j}}\right] \\
&+C_{1 \varepsilon} \frac{\varepsilon}{k}\left(G_{k}+C_{3 \varepsilon} G_{b}\right)-C_{2 \varepsilon} \rho \frac{\varepsilon^{2}}{k}+S_{\varepsilon},
\end{aligned}
$$

where $G_{k}$ is the generation of turbulence kinetic energy of the mean velocity gradients and, in a manner accordance with the Boussinesq hypothesis [29], $G_{k}$ is evaluated by the following first equation; $G_{b}$ is generated by turbulence kinetic energy due to buoyancy, and $Y_{M}$ is the parameter of the fluctuating dilatation and overall dissipation rate in compressible turbulence; those two parameters are usually set as zero when the fluid considered is incompressible; $C_{1 \varepsilon}, C_{2 \varepsilon}$, and $C_{3 \varepsilon}$ are constants, given usually by the following second equation(6); $\sigma_{k}$ and $\sigma_{\varepsilon}$ are the turbulence Prandtl numbers for $k$ and $\varepsilon$, given by default as (6); $S_{k}$ and $S_{\varepsilon}$ are user-defined source terms.

$$
\begin{aligned}
G_{k} & =\mu_{t} S^{2}, \\
\mu_{t} & =\rho C_{\mu}\left(\frac{k^{2}}{\varepsilon}\right), \\
S & \equiv \sqrt{2 S_{i j} S_{i j}}, \\
S_{i j} & =\frac{1}{2}\left(\frac{\partial u_{i}}{\partial x_{j}}+\frac{\partial u_{j}}{\partial x_{i}}\right), \\
C_{1 \varepsilon} & =1.44, \\
C_{2 \varepsilon} & =1.92, \\
C_{3 \varepsilon} & =\tanh \left|\frac{\nu}{u}\right|, \\
\sigma_{k} & =1.0, \\
\sigma_{\varepsilon} & =1.3 \\
C_{\mu} & =0.09 .
\end{aligned}
$$

2.1.2. RNG $k-\varepsilon$ Model. The RNG $k$ - $\varepsilon$ model, which was proposed by Yakhot and Orszag [31], was obtained through renormalization group theory. And the biggest change among the equations is the additional item $R_{\varepsilon}$ in $\mathcal{\varepsilon}$-equation to be more responsive to the effects of rapid strain and streamline curvature than the standard $k-\varepsilon$ model. The RNG $k-\varepsilon$ is given by

$$
\begin{aligned}
\frac{\partial}{\partial t}(\rho k) & +\frac{\partial}{\partial x_{i}}\left(\rho k u_{i}\right)=\frac{\partial}{\partial x_{j}}\left[\left(\mu+\frac{\mu_{t}}{\sigma_{k}}\right) \frac{\partial k}{\partial x_{j}}\right] \\
& +G_{k}-\rho \varepsilon+S_{k}, \\
\frac{\partial}{\partial t}(\rho \varepsilon) & +\frac{\partial}{\partial x_{i}}\left(\rho \varepsilon u_{i}\right)=\frac{\partial}{\partial x_{j}}\left[\left(\mu+\frac{\mu_{t}}{\sigma_{\varepsilon}}\right) \frac{\partial \varepsilon}{\partial x_{j}}\right] \\
+ & C_{1 \varepsilon} \frac{\varepsilon}{k} G_{k}-C_{2 \varepsilon} \rho \frac{\varepsilon^{2}}{k}-R_{\varepsilon}+S_{\varepsilon},
\end{aligned}
$$

where the additional item $R_{\varepsilon}$ is given by

$$
R_{\varepsilon}=\frac{C_{\mu} \rho \eta^{3}\left(1-\eta / \eta_{0}\right)}{1+\beta \eta^{3}} \frac{\varepsilon^{2}}{k},
$$

where $\eta=S k / \varepsilon, \eta_{0}=4.38, \beta=0.012$, and the other constants are given by

$$
\begin{aligned}
C_{1 \varepsilon} & =1.42, \\
C_{2 \varepsilon} & =1.68, \\
\sigma_{k} & =\sigma_{\varepsilon} \approx 1.393, \\
C_{\mu} & =0.0845 .
\end{aligned}
$$

2.1.3. Realizable $k-\varepsilon$ Model. Compared with standard $k-\varepsilon$ turbulence model, the realizable $k-\varepsilon$ model [32] has made some improvements and adjustments, containing an 
alternative formulation in the viscosity and modifying the $\mathcal{E}$-equation with deriving from an exact equation. And the modeled transport equations for $k$ and $\varepsilon$ are given as follows:

$$
\begin{aligned}
\frac{\partial}{\partial t}(\rho k) & +\frac{\partial}{\partial x_{j}}\left(\rho k u_{j}\right)=\frac{\partial}{\partial x_{j}}\left[\left(\mu+\frac{\mu_{t}}{\sigma_{k}}\right) \frac{\partial k}{\partial x_{j}}\right] \\
& +G_{k}-\rho \varepsilon+S_{k}, \\
\frac{\partial}{\partial t}(\rho \varepsilon) & +\frac{\partial}{\partial x_{j}}\left(\rho \varepsilon u_{j}\right)=\frac{\partial}{\partial x_{j}}\left[\left(\mu+\frac{\mu_{t}}{\sigma_{\varepsilon}}\right) \frac{\partial \varepsilon}{\partial x_{j}}\right] \\
& +\rho C_{1} S \varepsilon-\rho C_{2} \frac{\varepsilon^{2}}{k+\sqrt{\nu \varepsilon}}+S_{\varepsilon},
\end{aligned}
$$

where $C_{1}=\max [0.43, \eta /(\eta+5)], \eta=S(k / \varepsilon)$.

Here, $C_{\mu}$ is no longer constant in comparison with the standard and RNG $k-\varepsilon$ models; it is calculated as

$$
\begin{aligned}
C_{\mu} & =\frac{1}{A_{0}+\left(A_{S} k U^{*} / \varepsilon\right)}, \\
A_{0} & =4.04 \\
A_{S} & =\sqrt{6} \cos \phi, \\
U^{*} & =\sqrt{S_{i j} S_{i j}+\widetilde{\Omega}_{i j} \widetilde{\Omega}_{i j}}, \\
\phi & =\frac{1}{3} \cos { }^{-1}(\sqrt{6} W), \\
W & =S_{i j} S_{j k} S_{k i} / \widetilde{S}^{3}, \\
\widetilde{S} & =\sqrt{S_{i j} S_{i j}}, \\
\widetilde{\Omega}_{i j} & =\Omega_{i j}-2 \varepsilon_{i j k} \omega_{k}, \\
\Omega_{i j} & =\overline{\Omega_{i j}}-\varepsilon_{i j k} \omega_{k} .
\end{aligned}
$$

The constants in (11) and (12) are given by $C_{2}=1.9$, $\sigma_{k}=1.0$, and $\sigma_{\varepsilon}=1.2$.

2.2. Numerical Schemes and Settings. The case is in a Cartesian coordinate $(x, y)$ (Figure 1$)$. The $x$-axis is aligned with the inlet flow direction, and the $y$-axis is perpendicular to $x$ and is aligned with the height direction. Rectangular cylinders with various side ratios $(R=b / h)$ are exposed to a uniform stream velocity $U=10 \mathrm{~m} / \mathrm{s}$. The height of the box girder is $1 \mathrm{~m}$; thus $R e$ is $6.85 \times 10^{5}$.

An incompressible flow with constant fluid properties is assumed. All geometrical lengths are scaled with $h$. The normalized dimensions of the domain $\left(L_{U}, L_{D}, h\right.$, and $H$, seen in Figure 1) are selected as 12, 30, 1, and 20, respectively. The $R$ is selected as $0.1,0.2,0.3,0.4,0.5,0.62,0.7,0.8$, $0.9,1.0,1.5,2.0,2.8,3.0$, and 4.0.

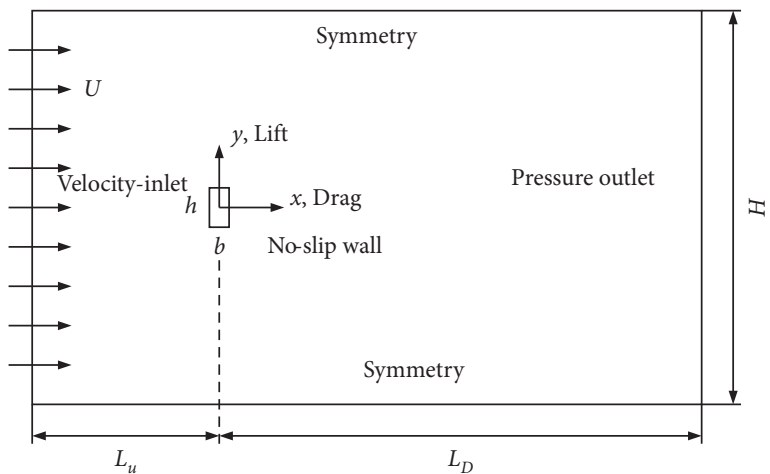

FIgURE 1: Computational domain and boundary conditions.

The boundary conditions used for the computations are applied as follows (seen in Figure 1): a uniform flow $(u=U$, $v=0$ ) is prescribed at the inlet boundary, the turbulence intensity is set as $I=5 \%$ (medium turbulence intensity), and the pressure is set as zero normal gradients. At the outlet, the velocity and turbulence fields $(k$ and $\varepsilon$ ) are specified as zero normal gradients, and the zero-pressure outlet boundary condition is used. No-slip conditions are prescribed at the surface of the cylinder; the pressure and turbulence fields are set as zero. Symmetry conditions with a frictionless wall $(\partial u / \partial y=0, v=0)$ are used at the upper and lower boundaries.

Mesh treatments are shown in Figure 2. The O-type grid was adopted for the simulations. The O-type grids were distributed around the cylinder with a distance of $5 \mathrm{~h}$, according to the hyperbolic tangent laws. Outside of the O-type grids, they were extended far away from the cylinder at a constant growth ratio. The Courant number (Co) was used to adjust the stability and convergence of the calculation. It is defined as $C o=U \Delta t / y_{0}$, where $y_{0}$ is the distance of the first gird to the wall of cylinder and $\Delta t$ is the time step. In this paper, through adjusting the time step, the Courant number was set as to be around 0.5 (Co $<1$ is required according to the criterion), and the calculation of each step could be completed in 20 iterations.

Numerical simulations had been performed by using the commercial software Fluent [33], which allows the analysis of flow around rectangles, by employing an ALE (Arbitrary Lagrangian-Eulerian) formulation based on a colocated finite-volume approach. The RANS-based modeling technique is well embedded in FLUENT. The scheme applied in the present study was the pressure implicit with splitting of operators (PISO). The solver was pressure based and transient. Gradient is discretized spatially by the least square cell based scheme. Pressure is dealt with a second order spatial discretization scheme and momentum is a second order upwind scheme. The first order implicit was used for time integration.

2.3. Definitions of Characteristic Parameters. The formula of Reynolds number and the Strouhal number is defined as follows: 


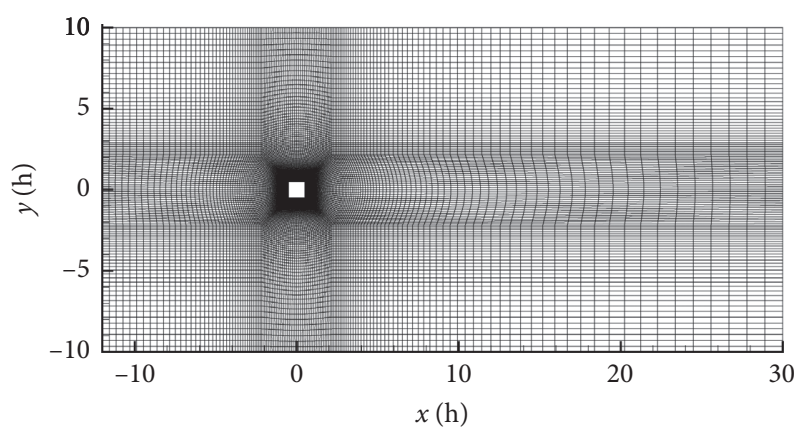

(a)

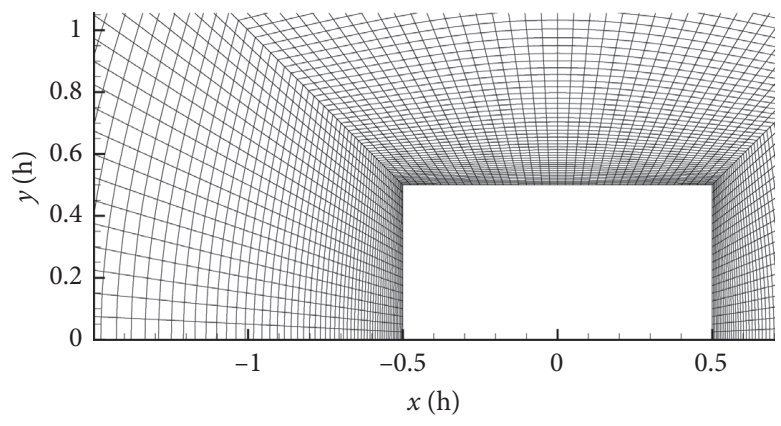

(b)

Figure 2: Grid distribution of the cylinder in O-block type. (a) Whole computational domain. (b) O-block type grids around the cylinder.

$$
\begin{aligned}
\mathrm{Re} & =\frac{U h}{v}, \\
\mathrm{St} & =\frac{f h}{U},
\end{aligned}
$$

where $f$ is the frequency of the vortex shedding.

The aerodynamic parameters of time-averaged drag and lift coefficient $\left(C_{d}\right.$ and $\left.C_{l}\right)$, the distribution of mean pressure coefficient $\left(C_{p}\right)$ around the cylinders, and the RMS value or fluctuation of lift coefficient $\left(C_{\text {lrms }}\right)$ are

$$
\begin{array}{r}
C_{d}=\frac{F_{d}}{1 / 2 \rho U^{2} h}, \\
C_{l}=\frac{F_{l}}{1 / 2 \rho U^{2} b}, \\
C_{p}=\frac{p-p_{\infty}}{1 / 2 \rho U^{2}}, \\
C_{l \mathrm{rms}}=\sqrt{\frac{\int_{0}^{T} C_{l}(t)^{2} \mathrm{~d} t}{T}},
\end{array}
$$

where $F_{d}$ and $F_{l}$ are the streamwise and cross-stream timeaveraged force components on the cylinders per unit length, respectively; $p$ and $p_{\infty}$ are the certain point of the cylinder surface and the reference pressure components of location, respectively; $C_{l}(t)$ is the instantaneous value of lift coefficient; $T$ is the fluctuation period.

\section{Results and Discussion}

3.1. Comparison with Different $k-\varepsilon$ Models. In order to study the effects of the three $k-\varepsilon$ turbulence models on the results, the square cylinder at $R e=6.85 \times 10^{5}$ was taken as an example for comparison and analysis.

Considering the fact that the accuracy and the efficiency of the results have a lot to do with the resolution of the grids, four grids with various distances $y_{0}$ were discussed. The details of grids and computational results are shown in Table 1 . The main aerodynamic integral parameters such as
$C_{d}, C_{l \mathrm{rms}}$, and $S t$ were presented and compared with available experimental $[1,10]$ and numerical results $[4,15,16,34]$. The results of $C_{d}$ by Lyn et al. [1], $C_{l \mathrm{rms}}$ by Rodi [34], and $S t$ by Lyn et al. [1] were chosen as the reference values; the relative deviations of all cases were shown in Table 1.

The cases in Table 1 with different grids were signed with G1 G4, and different turbulence models were signed with M1 M3. The minimum relative deviation of drag coefficient, fluctuation of lift coefficient, and Strouhal number were the cases M3G4, M3G4, and M1G1, respectively. The maximum relative deviation of them is M1G1 (or M1G3 and M1G4), M2G1, and M1G3. The detailed comparative analysis of the methods and grids is shown more intuitively in Figure 3.

The comparison of $C_{d}$ with different models is presented in Figure 3(a). For each turbulence model, the value of $C_{d}$ changes a little as $y_{0} / h$ ranging from 0.0005 to 0.002 , while the distinction between the three models at a certain $y_{0} / h$ is significantly different. The difference of the realizable $k-\varepsilon$ model with the reference values $[1,4,15]$ is the smallest; the minimum relative deviation is only $1 \%$ when $y_{0} / h$ is 0.002 . The result of RNG $k-\varepsilon$ model is slightly larger than the reference values, and the standard $k-\varepsilon$ model is smaller; the relative deviation of the standard $k-\varepsilon$ is the largest. Their minimum relative deviation is $19.5 \%$ and $10.5 \%$, respectively. Thus, for the prediction of $C_{d}$ among the three models, the realizable $k-\varepsilon$ is better than the others.

Figure 3 (b) presents the comparison of $C_{l \mathrm{rms}}$. The values of $C_{l \mathrm{rms}}$ for each model are almost unchanged when $y_{0} / \mathrm{h}$ increases, too. For every $y_{0} / h$, the RNG $k-\varepsilon$ model is always larger than the reference value $[4,15,34]$; the standard $k-\varepsilon$ model is smaller than the reference values. The realizable $k-\varepsilon$ model is slightly less than the reference values $[4,15]$ and nearly the same as Rodi's [34] result. The results of realizable $k-\varepsilon$ model are the best.

Figure 3(c) shows $S t$ of three models with $[1,4,15]$. The results change irregularly with the increase of $y_{0} / h$ in all three models, but it still could be observed that the realizable $k-\varepsilon$ model performs the best and the standard and RNG $k-\varepsilon$ perform the worst.

The distributions of $C_{p}$ on the surfaces of three models when $y_{0} / h=0.002$ are plotted in Figure 4. As shown in Figure $4, C_{p}$ on the front side of the surface (A-B) is positive and is in a good agreement with the experimental [35] and 


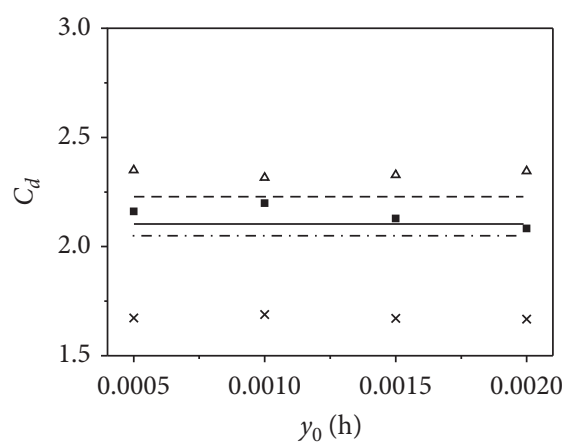

$\times$ Present-standard ke

$\Delta \quad$ Present-RNG ke

- Present-realizable ke

— EXP (Lyn et al. 1995)

- - LES (Sohankar 2006)

- - - RANS (Shimada et al. 2002)

(a)

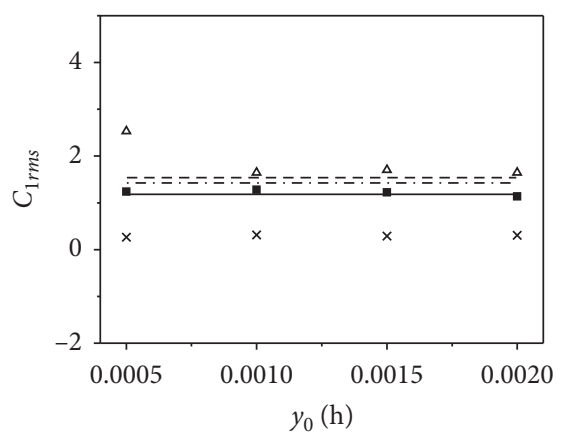

$\times \quad$ Present-standard ke

$\Delta \quad$ Present-RNG ke

- Present-realizable ke

- - LES (Sohankar, 2006)

... - RANS (Shimada, et al. 2002)

— RANS (Rodi et al. 1997)

(b)

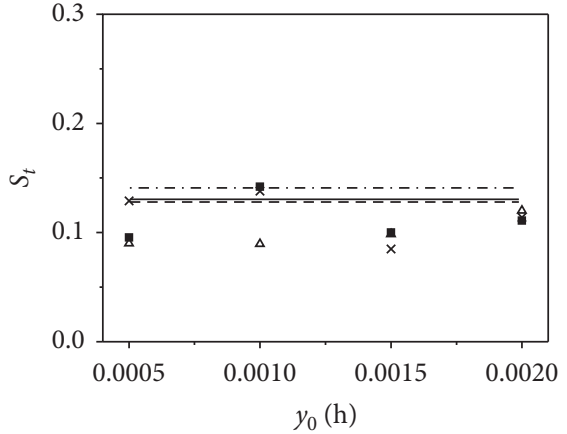

$\times \quad$ Present-standard ke

$\Delta \quad$ Present-RNG ke

- Present-realizable ke

— EXP (Lyn et al. 1995)

- - - LES (Sohankar 2006)

-. - RANS (Shimada et al. 2002)

(c)

Figure 3: Variation of aerodynamic characteristic $\left(C_{d}, C_{l \mathrm{rms}}\right.$, and $\left.S t\right)$ with different RANS models as $y_{0} / h$ ranging from 0.0005 to 0.0020 . (a) $C_{d}$ with different models. (b) $C_{l \mathrm{rms}}$ with different models. (c) St with different models.

TABLE 1: Comparison of integral parameters with different models.

\begin{tabular}{|c|c|c|c|c|c|c|c|c|c|}
\hline \multicolumn{2}{|c|}{ Case } & $y_{0} / h\left(\times 10^{-3}\right)$ & \multirow{2}{*}{$\frac{\operatorname{Re}\left(\times 10^{4}\right)}{68.5}$} & \multirow{2}{*}{$\begin{array}{c}C_{d} \\
1.67\end{array}$} & \multirow{2}{*}{$\begin{array}{c}\begin{array}{c}\text { Relative } \\
\text { deviation (\%) }\end{array} \\
20.5\end{array}$} & \multirow{2}{*}{$\begin{array}{c}C_{\text {lrms }} \\
0.02\end{array}$} & \multirow{2}{*}{$\begin{array}{c}\begin{array}{c}\text { Relative } \\
\text { deviation (\%) }\end{array} \\
98.3\end{array}$} & \multirow{2}{*}{$\begin{array}{c}\text { St } \\
0.129\end{array}$} & \multirow{2}{*}{$\begin{array}{c}\begin{array}{c}\text { Relative } \\
\text { deviation (\%) }\end{array} \\
2.3\end{array}$} \\
\hline \multirow{4}{*}{ Standard $k-\varepsilon$} & M1G1 & 0.5 & & & & & & & \\
\hline & M1G2 & 1.0 & & 1.69 & 19.5 & 0.31 & 73.5 & 0.138 & 4.5 \\
\hline & M1G3 & 1.5 & & 1.67 & 20.5 & 0.29 & 75.2 & 0.085 & 35.6 \\
\hline & M1G4 & 2.0 & & 1.67 & 20.5 & 0.30 & 74.4 & 0.114 & 13.6 \\
\hline \multirow{3}{*}{ RNG $k-\varepsilon$} & M2G1 & 0.5 & & 2.35 & 11.9 & 2.53 & 116.2 & 0.090 & 31.8 \\
\hline & M2G2 & 1.0 & & 2.32 & 10.5 & 1.65 & 41.0 & 0.090 & 31.8 \\
\hline & M2G3 & 1.5 & & 2.33 & 11.0 & 1.70 & 45.3 & 0.099 & 25.0 \\
\hline \multirow{5}{*}{ Realizable $k-\varepsilon$} & M2G4 & 2.0 & & 2.35 & 11.9 & 1.65 & 41.0 & 0.120 & 9.1 \\
\hline & M3G1 & 0.5 & & 2.16 & 2.9 & 1.24 & 6.0 & 0.096 & 27.3 \\
\hline & M3G2 & 1.0 & & 2.2 & 4.8 & 1.28 & 9.4 & 0.142 & 7.6 \\
\hline & M3G3 & 1.5 & & 2.13 & 1.4 & 1.23 & 5.1 & 0.100 & 24.2 \\
\hline & M3G4 & 2.0 & & 2.10 & 1.0 & 1.14 & 2.6 & 0.111 & 15.9 \\
\hline \multicolumn{3}{|c|}{ EXP(Norberg [10]) } & 1.3 & 2.16 & $2.8^{\mathrm{R}}$ & - & - & 0.132 & $15.9^{\mathrm{R}}$ \\
\hline \multicolumn{3}{|c|}{ EXP (Lyn et al. [1]) } & 2.14 & $2.1^{*}$ & - & - & - & $0.132^{*}$ & - \\
\hline \multicolumn{3}{|c|}{ LES (Sohankar [4]) } & 2.2 & 2.32 & $9.5^{\mathrm{R}}$ & 1.54 & $26.0^{\mathrm{R}}$ & 0.132 & $15.9^{\mathbf{R}}$ \\
\hline \multicolumn{3}{|c|}{ TL-K1 $k-\varepsilon$ (Rodi [34]) } & 2.2 & 2.0 & $5.0^{\mathrm{R}}$ & $1.17^{*}$ & - & 0.143 & $22.4^{\mathrm{R}}$ \\
\hline \multicolumn{3}{|c|}{ Modified $k-\varepsilon$ (shimada and Ishihara [15]) } & 2.2 & 2.05 & $2.4^{\mathrm{R}}$ & 1.43 & $20.3^{\mathbf{R}}$ & 0.141 & $21.3^{\mathbf{R}}$ \\
\hline \multicolumn{3}{|c|}{ SST k- $\omega$ (Tian et al. [16]) } & 2.14 & 2.06 & $1.9^{\mathrm{R}}$ & 1.49 & $23.5^{\mathrm{R}}$ & 0.138 & $19.6^{\mathbf{R}}$ \\
\hline
\end{tabular}

Values with * in bold were the reference values for relative deviation calculation and values with superscript ${ }^{\mathrm{R}}$ represent the relative deviation between M3G4 and the corresponding reference literature.

numerical $[15,36]$ results for all of models. On the sidesurface (B-C) and back surface (C-D), $C_{p}$ is negative, which indicates that the direction and components of the flow velocity have changed when flow passes by the cylinder and its kinetic energy has been consumed because of the viscous sublayer near the surfaces. So, the relative pressure declines. The predictions of $C_{p}$ with the three models are different: the standard $k-\varepsilon$ model underestimates the pressure (absolute value) on B-C-D sides, and the RNG and realizable $k-\varepsilon$ models show a good agreement with the reference studies $[15,35,36]$.
It can be seen that $C_{d}, C_{l \mathrm{rms}}$, and $C_{p}$ of realizable $k-\varepsilon$ model show a good agreement with the references compared to the other two models, while the relative deviation of $S t$ is still large. The difference is related to the fact that the RANS $k-\varepsilon$ models are two-dimensional simplified methods and the three-dimensional effect is ignored which has been discussed in [24-26]. For large structures in engineering practice, the drag force and the distribution of surface wind pressure are more important and need to be considered, so that the deviation is acceptable. 


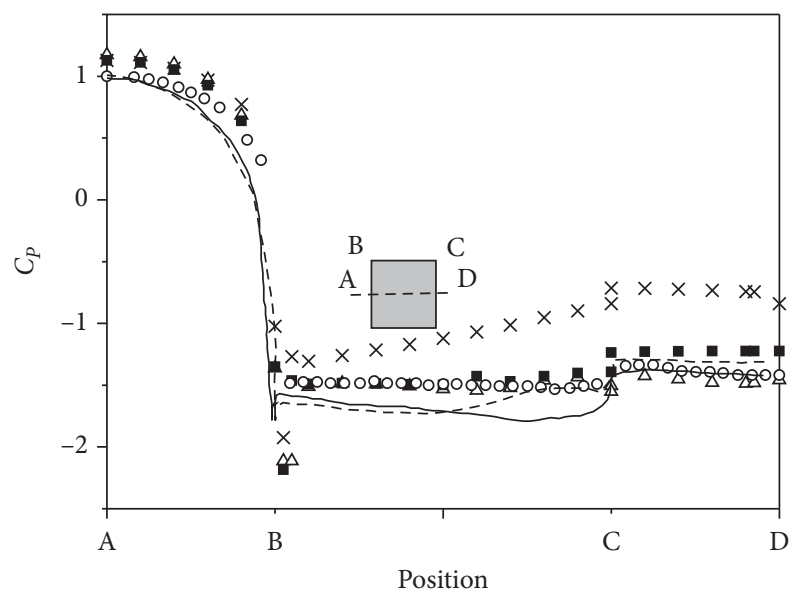

$\times \quad$ Present (standard ke)

$\Delta \quad$ Present (RNG ke)

- Present (realizable ke)

- $\operatorname{EXP}($ Lee, $\operatorname{Re}=1.76 \mathrm{e} 5,1975)$

- LES (Oka\&Ishihara, $\mathrm{Re}=4 \mathrm{e} 4,2009$ )

- - - Modified ke (Shimada et al., $\mathrm{Re}=2.2 \mathrm{e} 4,2002$ )

Figure 4: Distribution of $C_{p}$ around cylinder with different RANS models.

Overall, the time-averaged integral components change a little when $y_{0} / h$ increases except for St; thus the three turbulence models are not sensitive in grids. Among the three turbulence models, for the predicted values of $C_{d}$, $C_{\text {lrms }}, \mathrm{St}$, and $C_{p}$ on the surfaces, the realizable $k$ - $\varepsilon$ model's performance is always the best and the standard $k-\varepsilon$ model is the worst. As a result, the realizable $k-\varepsilon$ is the final model that we decided to choose, and the case M3G4 was taken as the reference for the rectangular cylinders' simulations in the following computations.

\subsection{Effect of Various $R$}

3.2.1. Aerodynamic Characteristics with Various $R$. Drag coefficient $\left(C_{d}\right)$ varying with different side ratios $(R)$ is shown in Figure 5(a). Compared with the related results $[5,10,12,17,20]$, the present predicted $C_{d}$ shows a good agreement with them. As side ratio $(R)$ grows up from 0.1, the drag coefficient $\left(C_{d}\right)$ increases and reaches its peak at $R \approx 0.62$ and then decreases with $R$ which continues to increase; finally $C_{d}$ changes a little after $R \geq 2.8$. The changes of $C_{d}$ at various $R$ can be explained as in Bearman and Trueman's [19] studies. When $R$ reaches its critical value (about 0.62 ), the base cavity gradually decreases due to the interaction of the separation shear layer near the surface of the cylinder, resulting in the maximum of $C_{d}$. When $R$ exceeds the critical value, with the side-surface increasing longer, the trailing vortex generated by the back corner of the cylinder makes $C_{d}$ smaller. But Nakamura and Ohya [8] explained it in another way. They claimed that the turbulence scale of the wake, the extra small-scale vortex, and the transverse vibration (sometimes the resonance) might be the main factor when $R$ reaches the critical value of 0.62 .
According to the trend of $C_{d}$, we divided the side ratios into three parts artificially: Regime I $(R \leq 0.62)$, Regime II $(0.62 R \leq 2.8)$, and Regime III $(R \geq 2.8)$. Regimes I and II are distinguished by the local maximum of the drag coefficient.

Figure 5(b) shows the base pressure coefficient $\left(-C_{p b}\right)$ at various $R$. The tendency of $-C_{p b}$ is the same as $C_{d}$. It goes up in Regime I, then goes down in Regime II, and finally becomes nearly stable in Regime III. The variation is also in a good agreement with the available results $[5,10,17]$.

The fluctuation of the lift coefficient $\left(C_{l \mathrm{rms}}\right)$ varying with $R$ is shown in Figure 5(c). Lack of enough experimental data and some typical published results with RANS and LES models $[15,17]$ were compared. The fluctuation of lift coefficient $C_{l \mathrm{rms}}$ increases with the increasing of $R$ in Regime I, then it decreases in Regime II, and next it increases suddenly at $R=2.8$ and finally declines again in Regime III. The predicted $C_{l \mathrm{rms}}$ is almost the same as the results of Shimada and Ishihara[15] and is also close to Sohankar's [17] results. However, the values of $C_{l \mathrm{rms}}$ in the present study are relatively smaller compared to the LES results [17] in Regimes II and III. According to Reynolds and Hussain's [37] studies, the fluctuation value consisted of periodic and stochastic fluctuation component, but RANS models ignore the stochastic turbulent fluctuation, and the proportion of stochastic fluctuation component depends a lot on $R$, so that the present results of $C_{l \mathrm{rms}}$ are always underestimated and show a difference at different $R$ [15]. As seen in Figure 5(c), the proportion of stochastic fluctuation in Regime II is larger than the other two regimes so that the present result of $C_{l \mathrm{rms}}$ is much lower than the LES results [17] in Regime II.

Figure 5(d) shows that the Strouhal number (St) changes with various $R$. The predicted $S t$ changes a little in Regime I and decreases with $R$ increases in Regimes II and III. But $S t$ presents a discontinuity at $R=2.8$; this is due to the reattachment of the vortex when $R$ is around 2.8. The result is just the same as the results of Nakaguchi et al. [5], Norberg [10], Okajima et al. [6, 12], and Sohankar [17].

Generally, the aerodynamic characteristics obtained from present RANS realizable $k-\varepsilon$ model are able to give reasonable predictions at $R=0.1-4.0$. The present results also indicate that the Reynolds dependence of the results has become much smaller for higher Reynolds numbers, which has been mentioned by Sohankar et al. $[4,17]$ and Nakamura and Ohya [8].

\subsubsection{Flow Field Characteristics with Various R. Figure 6} shows the distribution of the surface pressure coefficient $\left(C_{p}\right)$ along the front and back side of cylinders with different side ratios $(R=0.1,0.2,0.4,0.62,0.8,1.0,1.5,2.0,2.8$, and 3.0). The pressure coefficient $C_{p}$ on the front side for the side ratios collapses together, while the tendency of $C_{p}$ on the back side with various $R$ varies apparently, which suggests that the differences in drag are mainly due to the differences in the pressure distribution along the back side.

Meanwhile, the pressure distribution on the back side varies with different side ratios: the pressure changes apparently when $R<1.0$, especially when $R=0.62$, and it is almost constant when $R \geq 1.0$. As the regimes have been 


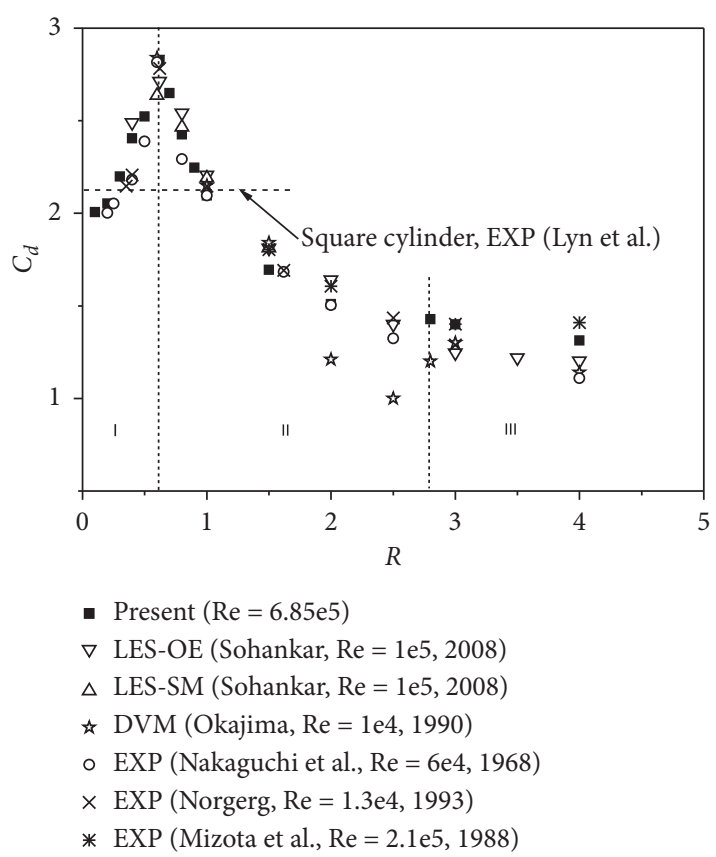

(a)

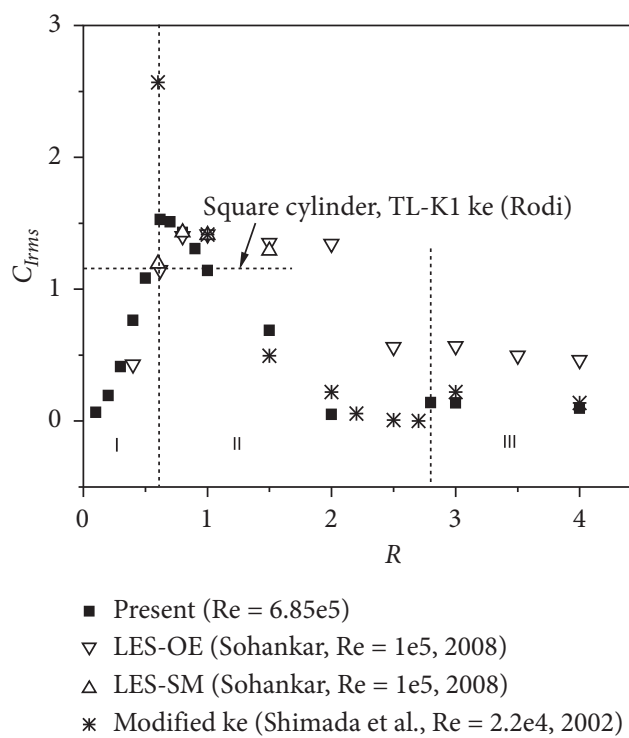

(c)

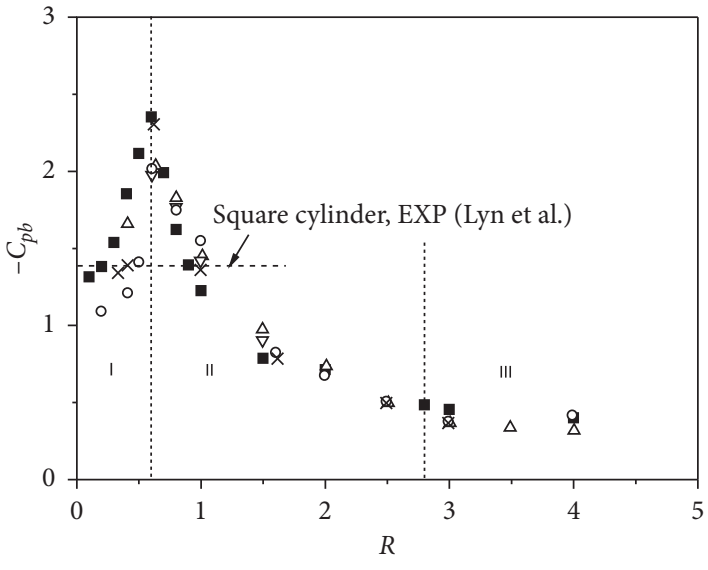

- Present $(\mathrm{Re}=6.85 \mathrm{e} 5)$

$\triangle$ LES-OE (Sohankar, $\mathrm{Re}=1 \mathrm{e} 5,2008)$

$\nabla$ LES-SM (Sohankar, Re $=1 \mathrm{e} 5,2008$ )

- EXP (Nakaguchi et al., $R e=6 e 4,1968$ )

$\times$ EXP (Norgerg, $\operatorname{Re}=1.3 \mathrm{e} 4,1993)$

(b)

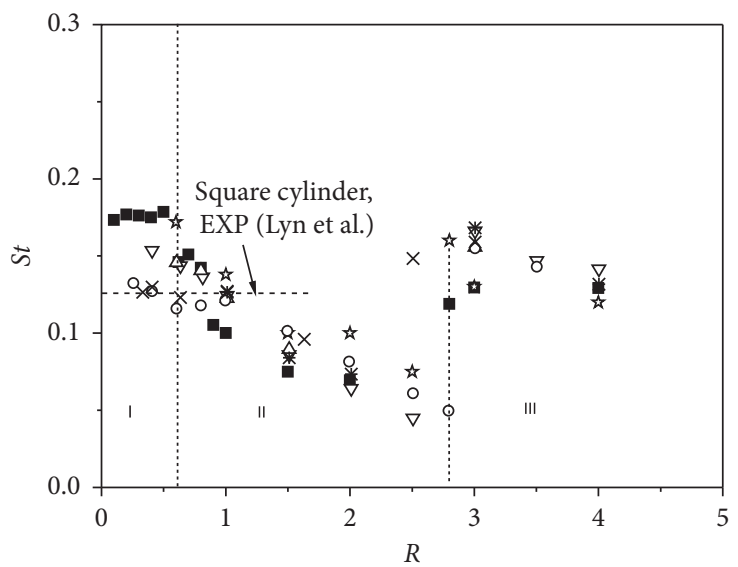

- Present $(\operatorname{Re}=6.85 \mathrm{e} 5)$

$\nabla$ LES-OE (Sohankar, Re $=1 \mathrm{e} 5,2008$ )

$\triangle$ LES-SM (Sohankar, Re $=1 \mathrm{e} 5,2008$ )

* DVM (Okajima, Re = 1e4, 1990)

- EXP (Nakaguchi et al., $R e=6 e 4,1968$ )

$\times$ EXP (Norberg, $\operatorname{Re}=1.3 \mathrm{e} 4,1993)$

* EXP (Okajima, $\mathrm{Re}=2 \mathrm{e} 4,1982$ )

(d)

Figure 5: Variation of aerodynamic parameters with different side ratios. (a) $C_{d}$. (b) $-C_{p b}$. (c) $C_{l \mathrm{rms}}$ (d) St.

defined above, the difference of $C_{p}$ varying along the back side mainly happens in Regimes I and II. These might come from the differences of vortex distributions of the wake with various $R$, concerning the fact that $C_{p}$ is actually generated by vortex shedding of the cylinder.

Figure 7 presents two parameters of the wake with different $R$ : recirculation length $\left(l_{R}\right)$ and the negative maximum velocity of back-flow ( $U_{B F}$, in absolute value). The recirculation length $l_{R}$ can be seen as the index of the wake distance and $U_{B F}$ indicates the strength of the wake. As seen in Figure 7, the values of $l_{R}$ and $U_{B F}$ varying with different $R$ can also be divided into three regimes as mentioned above. In Regime I, $l_{R}$ and $U_{B F}$ decrease when $R$ increases. In Regimes II and III, $l_{R}$ and $U_{B F}$ also increase with $R$ but grow up slowly in Regime III. The tendencies of $l_{R}$ and $U_{B F}$ varying with side ratios are just the opposite of $C_{d}$ and $-C_{p b}$.

Figure 8 shows the vorticity contours in the wake zone for various cylinders at $R=0.1,0.2,0.4,0.62,0.8,1.0,1.5,2.0$, 2.8 , and 3.0 as the lift coefficient reaches its maximum value. Because the size and position of the vortex in the wake zone 


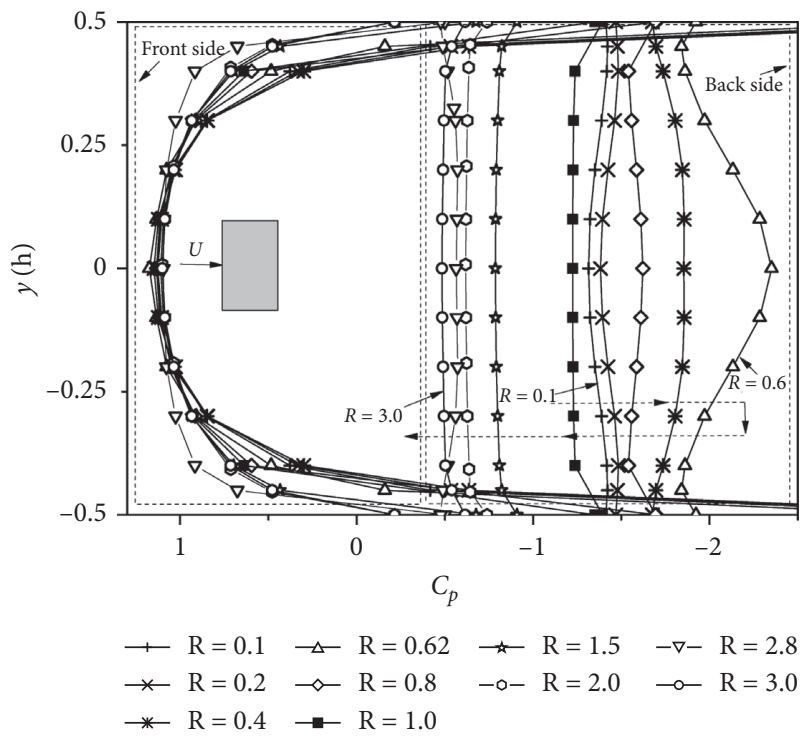

Figure 6: Distribution of $C_{p}$ around cylinder with various $R$.

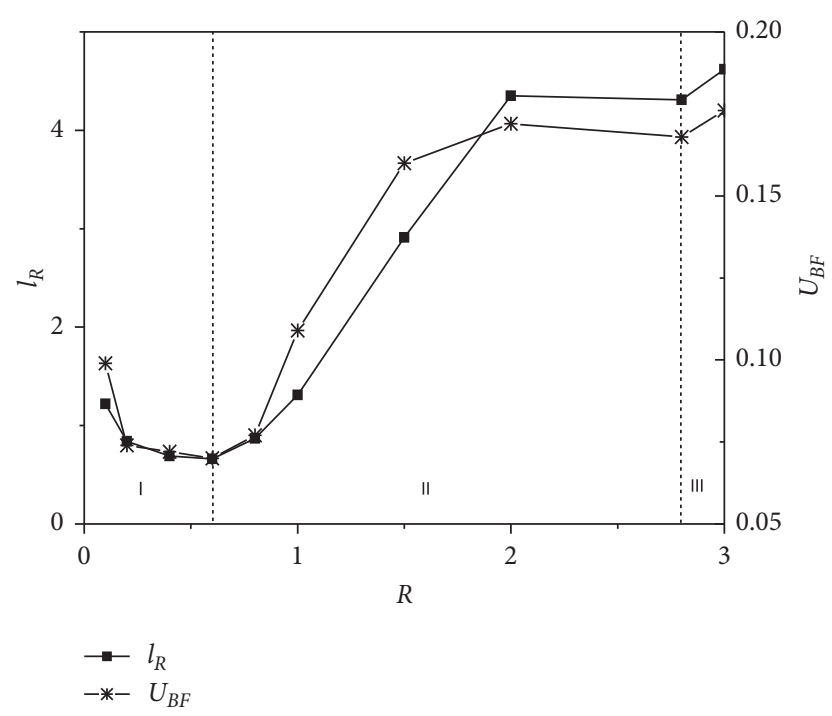

Figure 7: $l_{R}$ and $U_{B F}$ in the wake with various $R$.

would affect the pressure around the cylinders and the drag forces acting on the cylinder, it can be clearer to understand the aerodynamic characteristics mentioned above through the instantaneous vorticity contours. As can be seen in Figure 8, the fluid separates from the surface and forms the shear layer at the leading edge of the cylinders and then produces a base cavity near the back sides.

It is worth mentioning that the two-dimensional vorticity in Figure 8 is quite different from the three-dimensional vortex structure which was presented by Norberg [10], Sohankar et al. [4, 17], and Cao and Tamura [38]. As mentioned above, the exact value of turbulent fluctuation consisted of periodic and stochastic component, but the stochastic turbulent fluctuation is not modeled in RANS models. These stochastic perturbations, corresponding to the whole averaged flow fields, are small-scale eddies and are treated as a part of eddy viscosity [15]. Therefore, such smallscale eddies were not visible in the present flow patterns in Figure 8 . The present vorticity contours were more of a qualitative analysis of the flow field.

In Regime I, as seen in Figures 8(a)-8(d), the location of the generation of the vortex moves closer to back side when $R$ increases. Almost at $R=0.62$, the generation of the vortex becomes the strongest. Thus, $-\mathrm{Cpb}$ and $C_{d}$ reach their local maximum. When $R$ continues to grow up to Regimes II and III, as seen in Figures $8(e)-8(j)$, the location of the vortex begins to move further away from the cylinder, corresponding to the decline of $-C_{p b}$ and $C_{d}$. On the other hand, it could be observed that the flow separates from the front corners and is not reattached to the side faces in Regimes I and II (Figures 8(a)-8(i)). As seen in Figures 8(i) and 8(j), when side ratios are in Regime III, there is a flow reattachment phenomenon of wake vortex around the cylinder, which gives a good interpretation for the discontinuity of $S t$ and $C_{l \mathrm{rms}}$ in Figures 5(c) and 5(d).

In addition, the wake vortex has an important impact on the distributions of the pressure in the back side: the closer the vortex to the back side, the larger the pressure (in absolute) in the back side. Therefore, the distribution of the pressure in back side is changing when the vortex is closer to the cylinder at $R<1.0$, shown in Figure 6 , and pressure maintains almost constant when the vortex moves further away from the cylinder at $R \geq 1.0$. Particularly, when $R=0.62$, seen in Figure 8(d), the vortex attaches to the back side, and the pressure of the central position in the back side is the largest (in absolute value), because it just is located at the center of the vortex.

3.2.3. Discussion of the Three Regimes. Much attention has been paid to the effect of side ratios of rectangular cylinders on the aerodynamic characteristics. There are mainly two critical side ratios for aerodynamic parameters. The first one is located at $R=0.62$ for the maximum of mean drag and base pressure coefficient, and the other is for the discontinuity of Strouhal number when the side ratio equals 2.8 $[5,6,10,12,15-17,19]$.

However, there are little clear subregional discussion on the side ratios, and the characteristics of each region are only simply described. Nakaguchi et al. [5] listed numbers of experimental results at side ratios ranging from 0.1 to 4.0 , but correlation analysis and discussion were little. Okajima et al. $[6,7,12]$ discussed $C_{d}$, St, and flow patterns at $R=0.6-8.0$ containing wider range and got similar results. But some other characteristics such as $-C_{p b}$ and $C_{l \mathrm{rms}}$ were not analyzed, as well as the range of $R<0.6$. Norberg [10] conducted $C_{d}, C_{p}$, and $S t$ at $R=1,1.67,2.5$, and 3 at angles of attack $0^{\circ} \sim 90^{\circ}$; the series of side ratios are not enough to distinguish them. Shimada and Ishihara[15] researched the cylinders at $R=0.6 \sim 8.0$. Tian et al. [16] did not contain side ratios which are larger than 1.0.

Thus, three regimes at various side ratios were divided artificially in the present study which has been mentioned above. The main distinctions of three regimes are shown in Figure 9. Regimes I and II are divided on the basis of $C_{d}$ and 


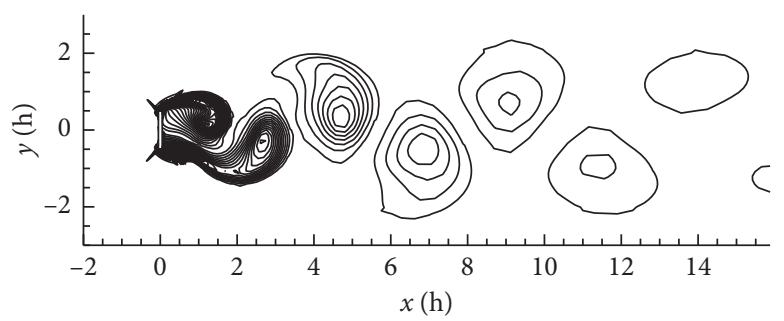

(a)

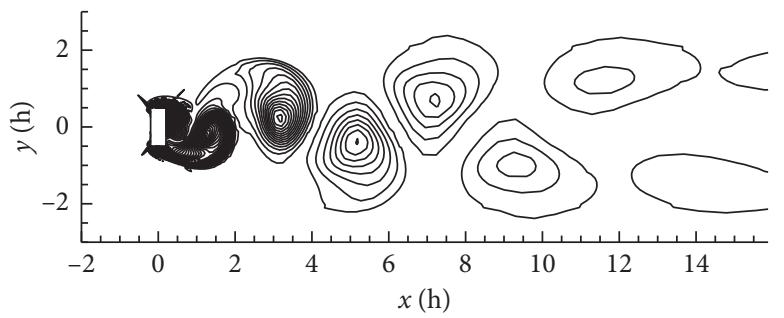

(c)

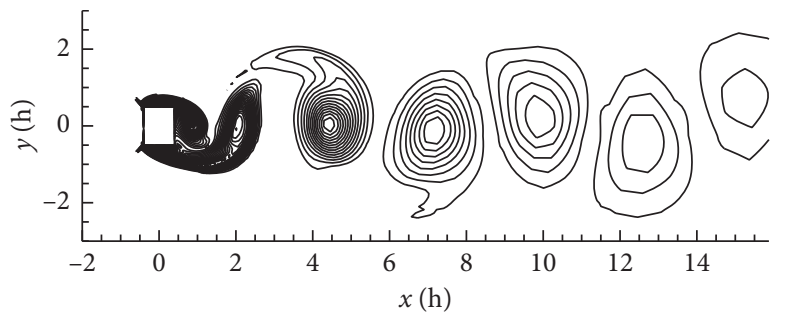

(e)

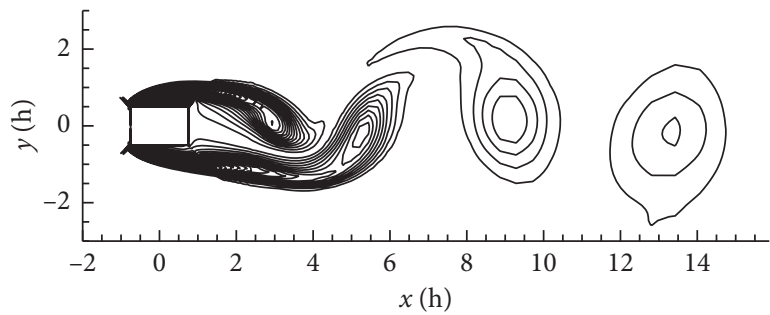

(g)

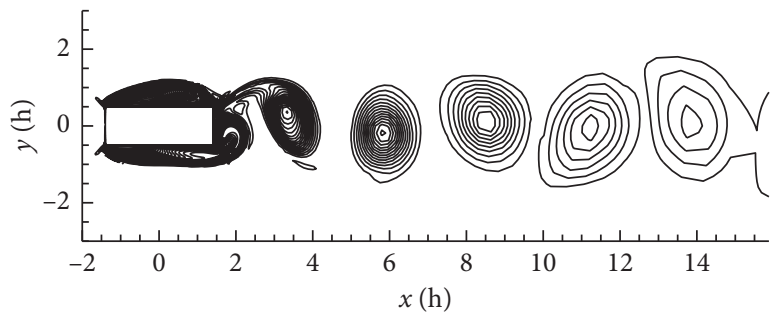

(i)

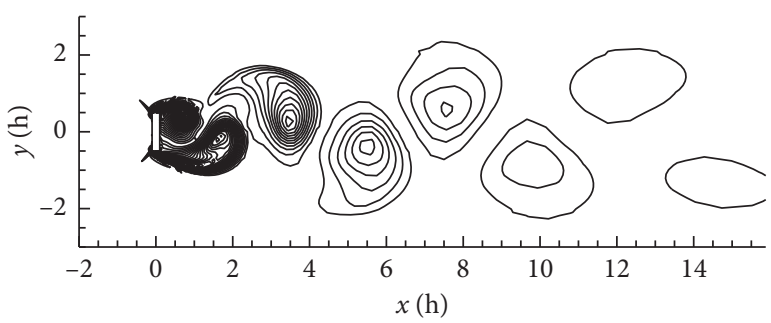

(b)

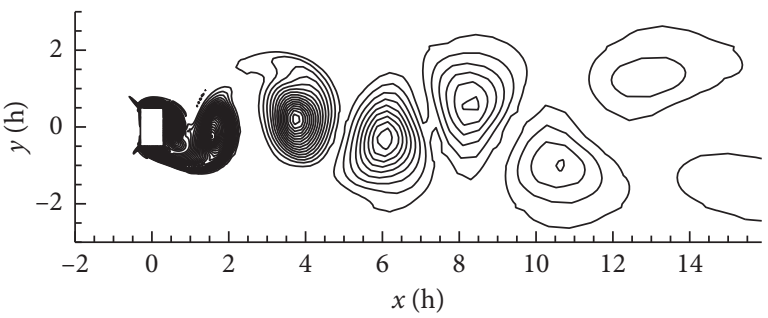

(d)

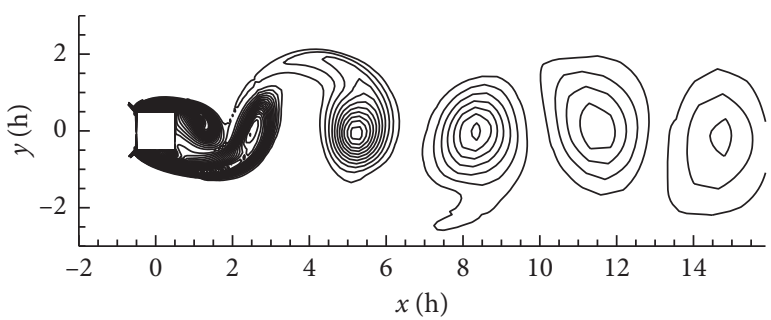

(f)

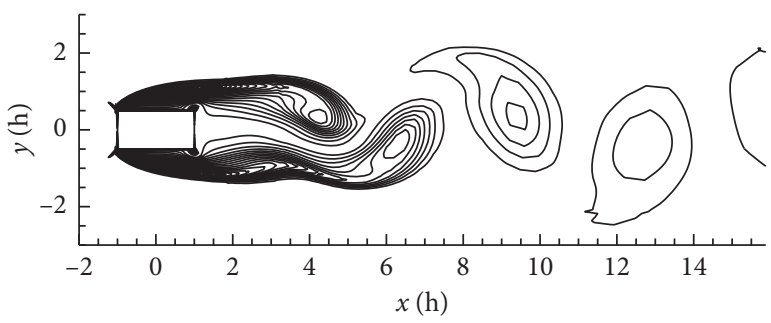

(h)

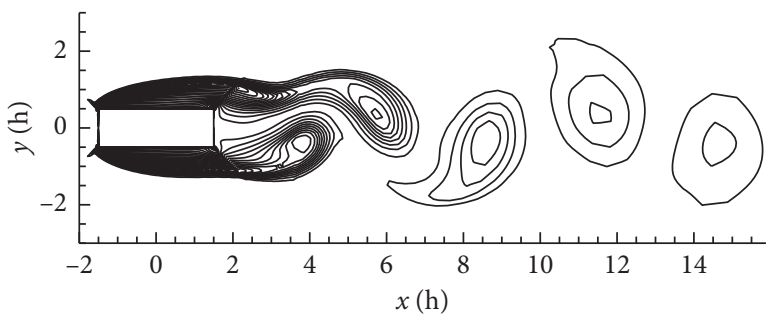

(j)

Figure 8: Vorticity contours with different side ratios when lift coefficient is maximum when $R=0.1,0.2,0.4,0.62,0.8,1.0,1.5,2.0,2.8$, and 3.0. (a) $R=0.1$. (b) $R=0.2$. (c) $R=0.4$. (d) $R=0.62$. (e) $R=0.8$. (f) $R=1.0$. (g) $R=1.5$. (h) $R=2.0$. (i) $R=2.8$. (j) $R=3.0$.

$-C_{p b}$ at $R=0.62$. Regimes II and III are distinguished by the discontinuity of $S t$ at $R=2.8$.

As shown in Figure 9, in Regime I, the aerodynamic parameters of $C_{d},-C_{p b}$, and $C_{\text {lrms }}$ increase when $R$ grows up and reach their local maximum at $R=0.62$. St keeps almost constant in Regime I. The pressure coefficient $C_{p}$ (in absolute value) on back side of different cylinders also shows similar increasing tendency, and the distribution of $C_{p}$ along the back side changes to be larger when $R$ increases. The wake characteristics of $l_{R}$ and $U_{B F}$ decrease with the increasing of $R$. The vortex in the wake (seen as the flow visualization in Figure 9) moves closer to the cylinder and becomes the closest at $R=0.62$.

In Regime II, $C_{d},-C_{p b}, C_{\text {lrms }}, S t$, and $C_{p}$ present a decreasing tendency with increase of $R$. The distribution of $C_{p}$ along the back side changes to be smaller when $R$ grows up 


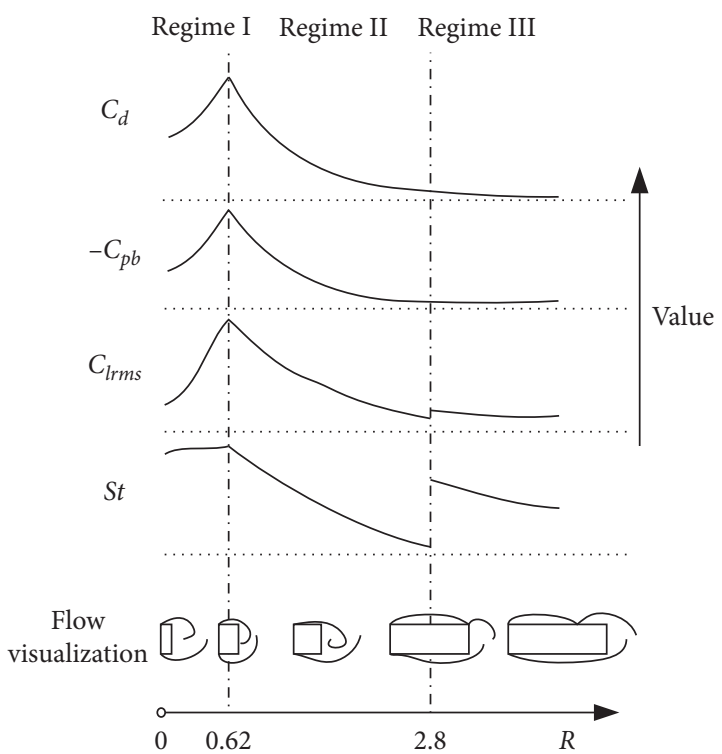

FIGURE 9: Schematic diagram of aerodynamic characteristics in three regimes.

and becomes almost constant at $R>1$.0. When $R$ increases, $l_{R}$ and $U_{B F}$ increase. The vortex in the wake begins to move away from the back side.

In Regime III, $C_{d},-C_{p b}$, and $C_{p}$ continue to decrease but change a little when $R$ increases. $C_{l r m s}$ and $S t$ present a sudden jump at $R=2.8$ and still show the same decreasing tendency at $R>2.8$. The recirculation length $l_{R}$ and the recirculation velocity $U_{B F}$ grow up with $R$ but change a little. The vorticity contours show a reattachment at $R \geq 2.8$, and the vortex moves further away from cylinder.

The characteristic of every regime is obvious and presents a difference with each other, and their boundaries represent a critical position. The division of the three regimes could give a clearer and more systematic cognition of the flow around rectangular cylinders. Moreover, the tendency of aerodynamic parameters varying with side ratios in high Reynolds number could give some guidance for practical engineering in design and manufacture. The drag force, base pressure, and the lift fluctuation appear to be at peak when side ratio equals 0.62 . Thus, this side ratio should be avoided to decline the structural drag force and to increase its stability in the wind fields. The discontinuity of Strouhal number and change of the lift coefficient fluctuation at side ratio of around 2.8 should also be noticed when referring to the vibrations of the structures. In other words, the side ratios near the boundaries of the three regimes should be taken into account in practical engineering.

\section{Conclusions}

In this paper, the flow around the rectangular cylinders with various side ratios for much higher Reynolds numbers $\left(R e=68.5 \times 10^{5}\right)$ was presented. Considering the cost and applying for the practical engineering, the two-dimensional RANS turbulence models were used for the computations. Three typical $k-\varepsilon$ turbulence models were compared and discussed firstly. Then, the aerodynamic characteristics such as the drag coefficient, the fluctuation of lift coefficient, the back pressure coefficient, and Strouhal number at various side ratios were presented and compared with the wind tunnel experimental results $[5,6,10,20]$ and numerical results $[15,17]$. Finally, the flow field characteristics around the cylinders were presented and illustrated in detail. The main conclusions in the research are summarized as follows:

(1) Three typical RANS $k-\varepsilon$ turbulence models were compared for the prediction of aerodynamic integral parameters. Through the comparisons of $C_{d}, C_{l \mathrm{rms}}$, $S t$, and $C_{p}$ over the cylinder, the realizable $k-\varepsilon$ model was found to perform the best and was finally chosen as the main method for the rectangular computations.

(2) According to the tendency of aerodynamic parameters varying with side ratios, three regimes (Regime I for $R \leq 0.62$; Regime II for $0.62 R \leq 2.8$; Regime III for $R \geq 2.8$ ) were divided. In Regime I, $C_{d},-C_{p b}$, and $C_{\text {lrms }}$ increase with the increasing of $R$ and reach their local maximum at $R=0.62$, and $S t$ changes a little; in Regime II, $C_{d},-C_{p b}, C_{l r m s}$, and $S t$ decrease with $R$; and, in Regime III, $C_{d},-C_{p b}, C_{l \mathrm{rms}}$, and $S t$ decrease less with $R$, but $C_{\text {lrms }}$ and $S t$ show a discontinuity at $R=2.8$.

(3) The flow field characteristics around the rectangular cylinders with various side ratios could also be distinguished with the three regimes. The contribution of $C_{p}$ along the back side presents a similar tendency as $C_{d}$ and- $C_{p b}$ in the three regimes. The wake fields parameters $l_{R}$ and $U_{B F}$ present an opposite tendency of $C_{d}$. The distinction of vortex in three regimes is presented with the visual vorticity contours. The closest vortex at $R=0.62$ and the reattachment of vortex at $R=2.8$ are shown more clearly and intuitively through the vorticity.

The two-dimensional RANS realizable $k-\varepsilon$ model adopted in this paper could give a reasonable prediction for the basic aerodynamic parameters and flow field characteristics with the comparison with published literatures. However, the derivative is large for the lift fluctuation and vortex shedding frequency when referring to the structural vibration and stability. Therefore, in the following study, the practicability and reliability of the model still need to be verified when it involves vortex-induced vibration, flutter, and other vibration problems.

\section{Data Availability}

The data used to support the findings of the study are available from the corresponding author upon request.

\section{Conflicts of Interest}

The authors declare that there are no conflicts of interest as regards the publication of this paper. 


\section{Acknowledgments}

This work was supported by the National Natural Science Foundation of China (NSFC) Project no. 51675450.

\section{References}

[1] D. A. Lyn, S. Einav, W. Rodi, and J.-H. Park, "A laser-doppler velocimetry study of ensemble-averaged characteristics of the turbulent near wake of a square cylinder," Journal of Fluid Mechanics, vol. 304, no. 1, pp. 285-319, 1995.

[2] S. C. Yen and C. W. Yang, "Flow patterns and vortex shedding behavior behind a square cylinder," Journal of Wind Engineering and Industrial Aerodynamics, vol. 99, no. 8, pp. 868-878, 2011.

[3] W. Rodi, J. H. Ferziger, M. Breuer, and M. Pourquie, "Status of large eddy simulation; results of a workshop," ASME Journal of Fluids Engineering, vol. 2, no. 119, pp. 248-262, 1997.

[4] A. Sohankar, "Flow over a bluff body from moderate to high reynolds numbers using large eddy simulation," Computers \& Fluids, vol. 35, no. 10, pp. 1154-1168, 2006.

[5] H. Nakaguchi, K. Hashimoto, and S. Muto, "An experimental study on aerodynamic drag of rectangular cylinders," Journal of the Japan Society for Aeronautical and Space Sciences, vol. 168 , no. 16 , pp. $1-5,1968$.

[6] A. Okajima, "Strouhal numbers of rectangular cylinders," Journal of Fluid Mechanics, vol. 123, 1982.

[7] A. Okajima, "Flow around a rectangular cylinder with a section of various width/height ratios," Wind Engineers, JAWE, vol. 1983, no. 17, pp. 1-19, 1983.

[8] Y. Nakamura and Y. Ohya, "The effects of turbulence on the mean flow past two-dimensional rectangular cylinders," Journal of Fluid Mechanics, vol. 149, no. 149, pp. 255-273, 1984.

[9] Y. Ohya, "Note on a discontinuous change in wake pattern for a rectangular cylinder," Journal of Fluids and Structures, vol. 8, no. 3, pp. 325-330, 1994.

[10] C. Norberg, "Flow around rectangular cylinders: pressure forces and wake frequencies," Journal of Wind Engineering and Industrial Aerodynamics, vol. 1-3, no. 49, pp. 187-196, 1993.

[11] S. Nakagawa, K. Nitta, and M. Senda, “An experimental study on unsteady turbulent near wake of a rectangular cylinder in channel flow," Experiments in Fluids, vol. 27, no. 3, pp. 284-294, 1999.

[12] A. Okajima, "Numerical simulation of flow around rectangular cylinders," Journal of Wind Engineering \& Industrial Aerodynamics, vol. 33, no. 1-2, pp. 171-180, 1990.

[13] A. Okajima, T. Nagahisa, and A. Rokugoh, "A numerical analysis of flow around rectangular cylinders," JSME International Journal. Ser. 2, Fluids Engineering, Heat Transfer, Power, Combustion, Thermophysical Properties, vol. 33, no. 4, pp. 702-711, 1990.

[14] M. V. Ian Taylor, "Prediction of unsteady flow around square and rectangular section cylinders using a discrete vortex method," Journal of Wind Engineering \& Industrial Aerodynamics, vol. 1-3, no. 82, pp. 247-269, 1999.

[15] K. Shimada and T. Ishihara, "Application of a modified k - $\varepsilon$ model to the prediction of aerodynamic characteristics of rectangular cross-section cylinders," Journal of Fluids and Structures, vol. 1, no. 16, pp. 465-485, 2002.

[16] X. Tian, M. C. Ong, J. Yang, and D. Myrhaug, "Unsteady RANS simulations of flow around rectangular cylinders with different aspect ratios," Ocean Engineering, vol. 58, no. 58, pp. 208-216, 2013.

[17] A. Sohankar, "Large eddy simulation of flow past rectangularsection cylinders: side ratio effects," Journal of Wind Engineering and Industrial Aerodynamics, vol. 96, no. 5, pp. 640-655, 2008.

[18] S. U. Islam, C. Y. Zhou, A. Shah, and P. Xie, "Numerical simulation of flow past rectangular cylinders with different aspect ratios using the incompressible lattice boltzmann method," Journal of Mechanical Science and Technology, vol. 26, no. 4, pp. 1027-1041, 2012.

[19] P. W. Bearman and D. M. Trueman, "An investigation of the flow around rectangular cylinders," Aeronautical Quarterly, vol. 23, no. 3, pp. 229-237, 1972.

[20] A. Okajima and T. Mizota, "Aerodynamic characteristics of fundamental structures (part 1)," Wind Engineers JAWE, vol. 1988, no. 36, 1988.

[21] W. Yupu, C. Wenming, R. DU, W. Shubiao, and D. Yong, "Bionic design method of crane box girder wind load reduction based on ostracion-cubicus," Journal of Southwest Jiaotong University, vol. 55, no. 3, pp. 664-671, 2020.

[22] A. Scarabino, J. Marañón Di Leo, J. S. Delnero, and F. Bacchi, "Drag coefficients and strouhal numbers of a port crane boom girder section," Journal of Wind Engineering and Industrial Aerodynamics, vol. 93, no. 6, pp. 451-460, 2005.

[23] S. Murakami, "Overview of turbulence models applied in CWE-1997," Journal of Wind Engineering and Industrial Aerodynamics, vol. 74-76, no. 98, pp. 1-24, 1998.

[24] K. Hanjalic, "Will RANS survive LES? A view of perspectives," Journal of Fluids Engineering, vol. 127, no. 127, p. 831, 2005.

[25] F. R. Menter and R. Florian, "Review of the shear-stress transport turbulence model experience from an industrial perspective," International Journal of Computational Fluid Dynamics, vol. 23, no. 4, pp. 305-316, 2009.

[26] B. Blocken, "LES over RANS in building simulation for outdoor and indoor applications: a foregone conclusion?" Building Simulation, vol. 11, no. 5, 2018.

[27] D. Sun, J. S. Owen, and N. G. Wright, "Application of the k- $\omega$ turbulence model for a wind-induced vibration study of $2 \mathrm{D}$ bluff bodies," Journal of Wind Engineering and Industrial Aerodynamics, vol. 97, no. 2, pp. 77-87, 2009.

[28] C. Mannini, A. Šoda, and G. Schewe, "Unsteady RANS modelling of flow past a rectangular cylinder: investigation of Reynolds number effects," Computers \& Fluids, vol. 39, no. 9, pp. 1609-1624, 2010.

[29] J. O. Hinze, Turbulence, McGraw-Hill Publishing Co, New York, NY, USA, 1975.

[30] B. E. Launder and D. B. Spalding, Lectures in Mathematical Model of Turbulence, Academic Press, London, England, UK, 1972.

[31] V. Yakhot and O. A. Orszag, "Renormalization group analysis of turbulence, I. Basic theory," Journal of Scientific Computing, vol. 1, no. 1, pp. 3-51, 1986.

[32] T.-H. Shih, W. W. Liou, A. Shabbir, Z. Yang, and J. Zhu, “A new $\mathrm{k}-\epsilon$ eddy viscosity model for high reynolds number turbulent flows," Computers \& Fluids, vol. 24, no. 3, pp. 227-238, 1995.

[33] F. Inc, Fluent 6.3 User's Guide, Fluent Inc, Lebanon, NH, USA, 2006.

[34] W. Rodi, "Comparison of LES and RANS calculations of the flow around bluff bodies," Journal of Wind Engineering and Industrial Aerodynamics, vol. 69-71, pp. 55-75, 1997.

[35] B. E. Lee, "The effect of turbulence on the surface pressure field of a square prism," Journal of Fluid Mechanics, vol. 69, no. 2, pp. 263-282, 1975. 
[36] S. Oka and T. Ishihara, "Numerical study of aerodynamic characteristics of a square prism in a uniform flow," Journal of Wind Engineering and Industrial Aerodynamics, vol. 97, no. 11-12, pp. 548-559, 2009.

[37] W. C. Reynolds and A. K. M. F. Hussain, "The mechanics of an organized wave in turbulent shear flow. Part 3. theoretical models and comparisons with experiments," Journal of Fluid Mechanics, vol. 54, no. 2, pp. 263-288, 1972.

[38] Y. Cao and T. Tamura, "Large-eddy simulations of flow past a square cylinder using structured and unstructured grids," Computers \& Fluids, vol. 137, pp. 36-54, 2016. 